\title{
CT Angiography Findings in Carotid Blowout Syndrome and Its Role as a Predictor of 1-Year Survival
}

\author{
C.-W. Lee, C.-Y. Yang, Y.-F. Chen, A. Huang, Y.-H. Wang, and H.-M. Liu
}

\begin{abstract}
BACKGROUND AND PURPOSE: Carotid blowout is a serious late complication of prior treatment of advanced head and neck cancer. We evaluate the efficacy of CTA in the diagnosis of impending carotid blowout syndrome in patients with head and neck cancer, and its capability to predict clinical outcome.
\end{abstract}

MATERIALS AND METHODS: The clinical data of 29 patients with impending carotid blowout who underwent CTA were collected and analyzed. Imaging signs included tissue necrosis, exposed artery, viable perivascular tumor, pseudoaneurysm, and contrast extravasation. DSA was obtained in 20 patients. One-year outcomes were compared based on management.

RESULTS: The most common CTA finding was necrosis (94\%), followed by exposed artery (73\%), viable tumor (67\%), pseudoaneurysm (58\%), and contrast extravasation (30\%). Exposed artery, pseudoaneurysm, and contrast extravasation were the 3 CTA findings related to outcomes. All of the pseudoaneurysm and contrast extravasation cases were associated with an exposed artery. An exposed artery was the most important prognostic predictor and could not be diagnosed on DSA. Patients without the 3 findings on CTA (group 1) had the best survival rate at 1-year follow-up, followed by patients with the 3 findings treated immediately by permanent artery occlusion (group 2). Patients with the 3 findings who had no immediate treatment (group 3$)$ had the worst outcomes $(P<.001$ in group 1 vs group 3 and group 2 vs group $3 ; P=.056$ group 1 vs group 2$)$.

CONCLUSIONS: CTA, with its ability to diagnose an exposed artery compared with DSA, may offer important management and prognostic information in patients with impending carotid blowout.

ABBREVIATIONS: $C B S=$ carotid blowout syndrome; $\mathrm{PAO}=$ permanent arterial occlusion

C arotid blowout syndrome (CBS) is defined as rupture of the carotid artery and its branches and is a serious complication after treatment of advanced head and neck cancer. Potential causes of CBS include radical resection, radiation therapy and radiation necrosis, carotid exposure, wound infection, pharyngocutaneous fistula, and recurrent or persistent carcinoma. ${ }^{1}$ The overall incidence of carotid blowout after neck dissection has been reported to be as high as $4.3 \%$, and the risk is increased another

Received April 28, 2013; accepted after revision June 28, 2013.

From the Department of Medical Imaging and Radiology (C.-W.L., C.-Y.Y., Y.-F.C., Y.-H.W., H.-M.L.), Hospital and Medical College, National Taiwan University, Taipei, Taiwan; and Research Center for Adaptive Data Analysis (A.H.), National Central University, Jhongli City, Taiwan.

The work of A. Huang was supported by a grant from the National Science Council of Taiwan.

Please address correspondence to Hon-Man Liu, MD, Department of Medical Imaging, National Taiwan University Hospital, No. 7, Chung-Shan South Rd, Taipei, Taiwan, ROC 100; e-mail: hmliu@ntu.edu.tw

- Indicates open access to non-subscribers at www.ajnr.org

http://dx.doi.org/10.3174/ajnr.A3716 7.6-fold with further radiation therapy. ${ }^{2}$ CBS typically occurs $2-20$ years after surgery or radiation therapy, ${ }^{3,4}$ and average estimates of cumulative neurologic morbidity and mortality are above $60 \%$ and $40 \%$, respectively, in patients with CBS. ${ }^{5} \mathrm{CBS}$ can be categorized into 1 of 3 categories: threatened, impending, and acute carotid blowout. ${ }^{1}$ Threatened carotid blowout is defined as physical examination or imaging results that suggest inevitable hemorrhage from 1 of the carotid arteries or its branches if no action is taken. Impending carotid blowout (also called sentinel hemorrhage) is defined as transient hemorrhage that resolves spontaneously or with packing or pressure. Acute carotid blowout represents hemorrhage that cannot be controlled by packing or pressure. ${ }^{1}$ Surgical management of carotid blowout is usually technically difficult and is associated with high morbidity and mortality rates. ${ }^{1,2,6,7}$ After surgical ligation or permanent arterial occlusion (PAO) of the carotid artery, the incidence of immediate or delayed cerebral ischemic complications can be as high as $15 \%-$ $20 \% .^{7,8-12}$ The complication rate of a balloon occlusion test before $\mathrm{PAO}$ of the carotid artery is reported to be as high as $3.2 \%$, 
and it may be even higher in fragile postirradiation vessels. ${ }^{13}$ Delayed ischemia after passing the balloon occlusion test is yet another concern. ${ }^{10,14,15}$ Stent-graft deployment, with or without coiling, is another endovascular treatment of CBS. Stent-grafting can preserve the affected carotid flow but has a high rate of early and delayed complications. ${ }^{16-19}$ No significant difference in short-term outcome between stent-graft deployment and PAO has been reported, ${ }^{20}$ and long-term results have not been reported. ${ }^{17}$

CTA has become widely available and is sensitive and specific in the detection of hemorrhagic vascular disorders such as aneurysms, arteriovenous malformations, dural arteriovenous fistulas, and intracranial dissections. Contrast extravasation on CTA predicts hematoma expansion, mortality, and clinical outcome in primary intracerebral hemorrhage. ${ }^{21-26}$ To our knowledge, there have been no past reports about the use of CTA in the diagnosis of CBS or as an outcome predictor. The aim of our study was to evaluate the efficacy of CTA in the diagnosis of impending CBS, and its capability to predict clinical outcome after management.

\section{MATERIALS AND METHODS}

This retrospective study was approved by our institutional review board. We retrieved and reviewed the records of patients with head and neck bleeding treated at our institution from July $2010-$ October 2011. Patients were included if they had a history of head and neck cancer and were treated with surgery and/or radiation and/or chemotherapy, and had acute hemorrhage controlled with pressure or packing (impending CBS). ${ }^{1}$ Patients with bleeding that resolved spontaneously or with acute massive bleeding that needed immediate resuscitative therapy or embolization would not have undergone CTA at our institution and were excluded. Patients with bleeding that resolved spontaneously were closely observed for 24 hours, and CTA was performed if rebleeding occurred. The CTA findings, endovascular treatment, and outcomes were recorded and analyzed.

\section{CTA Imaging and Evaluation}

CTA was performed with a single 64-detector CT scanner (LightSpeed VCT; GE Healthcare, Milwaukee, Wisconsin). The routine CTA protocol for head and neck bleeding included scanning 3 phases: the precontrast phase, the arterial phase, and the venous phase. The scanning protocol was as follows: $64 \times 0.625$ collimation, 0.516 pitch, 0.4 -second rotational time, and $100 \mathrm{kVp} ; 200$ $\mathrm{mA}$ was used for precontrast studies, and $400 \mathrm{~mA}$ was used for arterial-phase and venous-phase studies. The coverage was from the aortic arch to the lateral ventricle level. Dual injectors were used. We measured the time of peak enhancement at the level of the aorta first with the bolus tracing method. A total of $12 \mathrm{~mL}$ of low-osmolarity contrast material was injected, followed by $16 \mathrm{~mL}$ of normal saline at a rate of $4 \mathrm{~mL} / \mathrm{s}$, which was scanned at a 2 -second interval at the level of the ascending aorta. A total of $60 \mathrm{~mL}$ of contrast material followed by $35 \mathrm{~mL}$ of normal saline at a rate of 4 $\mathrm{mL} / \mathrm{s}$ was injected for the arterial-phase scan after a delay. The venous phase was obtained 30 seconds after the arterial phase. The acquired images were reconstructed into axial, coronal, and sagittal images with $0.625-\mathrm{mm}$ section thickness and stored. Maximal intensity projection images were reconstructed from sub- tracted arterial-phase images. An experienced radiologist performed DSA with a biplane angiographic unit. When CTA indicated a treatment target, DSA was performed on the target vessel first. The vertebral arteries and contralateral common carotid artery were checked after PAO. Routinely, a $5 \mathrm{~F}$ catheter and low-osmolarity contrast medium were used, and filming was performed at a frame rate of 4 per second. The DSA was used as the reference standard for the diagnosis of pseudoaneurysm and contrast extravasation in CBS. 3D rotational angiography was not performed in CBS in order to save time. All CTA images and DSA images were independently and retrospectively evaluated by 2 experienced neuroradiologists (C.-W.L. and H.-M.L. with 8 and 25 years of experience, respectively). The CTA and DSA interpretations were done separately, and readers were blinded to the result of the other study. The CTA and DSA images were evaluated for the following characteristics: 1) viable perivascular tumor, which was defined as any enhancing soft tissue on CTA or tumor blush anywhere on DSA; 2) tissue or tumor necrosis, which was defined as an area with lack of contrast enhancement in the soft tissues of the neck or tumor mass, adjacent to the suspect vessel; 3) contrast extravasation, which was defined as leakage of contrast medium, especially during the venous phase; 4) pseudoaneurysm, which was defined as a focal bulging of more than $30 \%$ of the parent arterial diameter; and 5) exposed artery, which was defined as more than half of the arterial circumference involved by necrotic tissue.

Interpretation was performed by use of the following sequence. MIP images were used to look for pseudoaneurysm and extravasation first. Once contrast extravasation was found, the bleeding point was detected by checking the sequential changes in the arterial- and venous-phase images. Checking the necrotic margin for identification of small pseudoaneurysms or exposed arteries was particularly important. Viable tumor and necrosis were reported as simply present or absent. Long segmental exposed arterial branches were defined as a single exposed artery. Pseudoaneurysms in a segmental exposed artery were counted as a single pseudoaneurysm. If there were multiple pseudoaneurysms or exposed arteries, they were reported separately. If there was disagreement between the 2 readers, a consensus decision was made after discussion.

\section{Patient Treatment}

At our institute, the choice of treatment for impending CBS was PAO without a balloon occlusion test. Platinum coils were the primary embolic material, and $n$-butyl-2-cyanoacrylate was used only in small external carotid artery branch lesions. To save time and avoid the danger of worse bleeding by using heparin, we did not perform the balloon occlusion test before PAO. We did not treat the impending CBS with stent-graft deployment. Long-term results of treatment with a stent graft in impending CBS have not been investigated and are therefore unknown. ${ }^{16-19}$ Perioperative use of antiplatelets and anticoagulants in patients undergoing stent-graft treatment was another concern. The timing of endovascular intervention was dependent on the clinical presentation, CTA findings, and patient and family decision. If a patient refused endovascular treatment, palliative treatment was given. Palliative treatment of patients with impending CBS who declined aggres- 

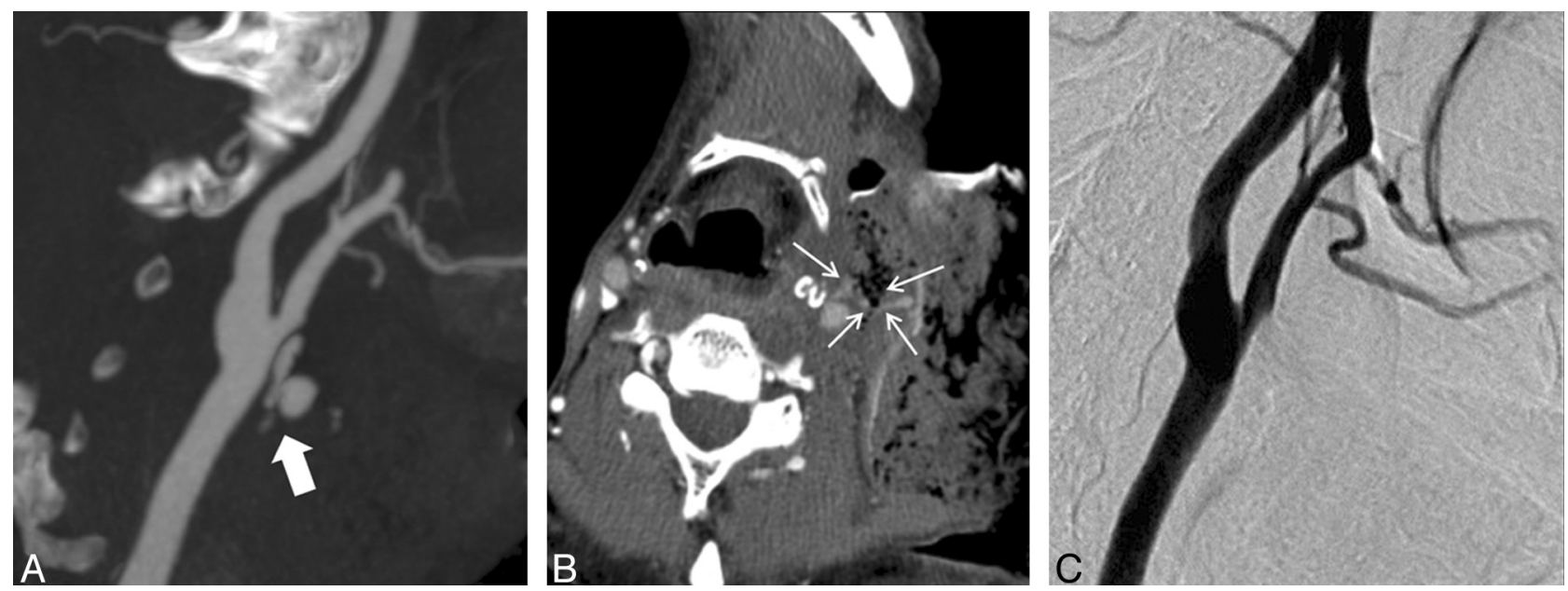

FIG 1. A 52-year-old man with a history of oropharyngeal cancer. A, MIP CTA imaging performed after bleeding was controlled by local packing shows contrast extravasation (arrow) from the common carotid artery near the bifurcation. B, Source image shows an exposed common carotid artery (arrows) surrounded by necrosis. C, DSA done immediately after CTA does not show contrast extravasation. However, the possibility of a further tamponade effect after CTA cannot be excluded in this case.

sive treatment included continuous pressure or packing, blood transfusion, fluid supplement, and tracheostomy if necessary.

\section{Patient Outcomes}

Patients were evaluated for at least 1 year after initial CTA or at the last hospital visit unless death occurred. We divided the patients into 3 groups according to the first CTA findings and treatment. Patients were categorized into group 1 if the CTA did not show pseudoaneurysm, extravasation, or an exposed artery, and they were treated conservatively. Patients were categorized as group 2 if the CTA demonstrated a pseudoaneurysm, extravasation, or an exposed artery, and they underwent immediate endovascular treatment. Patients were categorized as group 3 if the CTA demonstrated a pseudoaneurysm, extravasation, or an exposed artery, and they received no or delayed endovascular treatment.

\section{Statistical Analysis}

The adjusted Wald method was used to estimate the 95\% CIs of the observed rates. The $\kappa$ statistic was used to analyze the interobserver reliability. A value of $0.61-0.80$ implied substantial agreement, and a value of 0.81-1.00 implied almost perfect agreement. The Kaplan-Meier survival curve with the pair-wise log-rank test was used to assess the difference of survival among the 3 groups. Survival was considered statistically different if $P<.05$.

\section{RESULTS}

From July 2010-October 2011, a total of 29 patients (2 women and 27 men) with a mean age of 53.5 years (age range, 38-76 years) were treated for impending CBS and were included in this study. Eighteen patients had received a radical neck dissection, 25 had received radiation therapy, 6 had received a second course of radiation therapy, 22 had received chemotherapy, and 1 had received photodynamic therapy. Four patients had a second CTA for another bleeding episode 52 to 162 days after the first CTA. Twenty patients had DSA after the first CTA, and 3 patients underwent DSA after the second CTA. In total, 33 CTA examinations were available for evaluation.

\section{CTA Imaging and Evaluation}

The most common CTA imaging finding was necrosis (94\%), followed by an exposed artery (73\%), viable tumor (67\%), pseudoaneurysm (58\%), and contrast extravasation (30\%) (Figs 1 and 2). Contrast extravasation was more commonly found on the second CTA. The prevalence of image findings is listed in Table 1. Interobserver reliability showed almost perfect agreement regarding viable tumor, pseudoaneurysm, contrast extravasation, and exposed artery, and substantial agreement with respect to necrosis (Table 2). All pseudoaneurysms and contrast extravasation were associated with an exposed artery.

\section{DSA and Management}

Twenty-three DSA examinations were performed in 20 patients: 16 examinations were done within 6 hours after CTA, 6 were performed between 6 and 24 hours after CTA, and 1 was performed more than 24 hours after CTA. Two patients with a total of 3 pseudoaneurysms refused to undergo DSA after CTA. Three patients did not show evidence of pseudoaneurysm or extravasation on both the first CTA and DSA, but in 1 a pseudoaneurysm was identified on the second CTA and DSA that were performed 90 days later. The diagnostic performance of CTA compared with DSA in the detection of pseudoaneurysm and contrast extravasation was excellent. Comparison of both DSA and CTA results is shown in Table 3. Only 1 pseudoaneurysm was identified on DSA that was not seen on CTA; this occurred in a patient who had a delayed DSA performed after rebleeding.

Four patients had exposed arteries (including 1 patient with 2 exposed arteries) and refused endovascular management ( 3 patients at the first episode of bleeding and 1 patient at the second). One patient who received PAO died 63 days later as a result of rebleeding, at which time he declined further treatment. Two patients with exposed arteries on CTA did not undergo embolization immediately because their bleeding stopped before CTA, but massive bleeding subsequently occurred. Although embolizations were performed after resuscitation, they died at 7 and 18 days, respectively, after PAO. Another 2 patients had cardiac arrest be- 

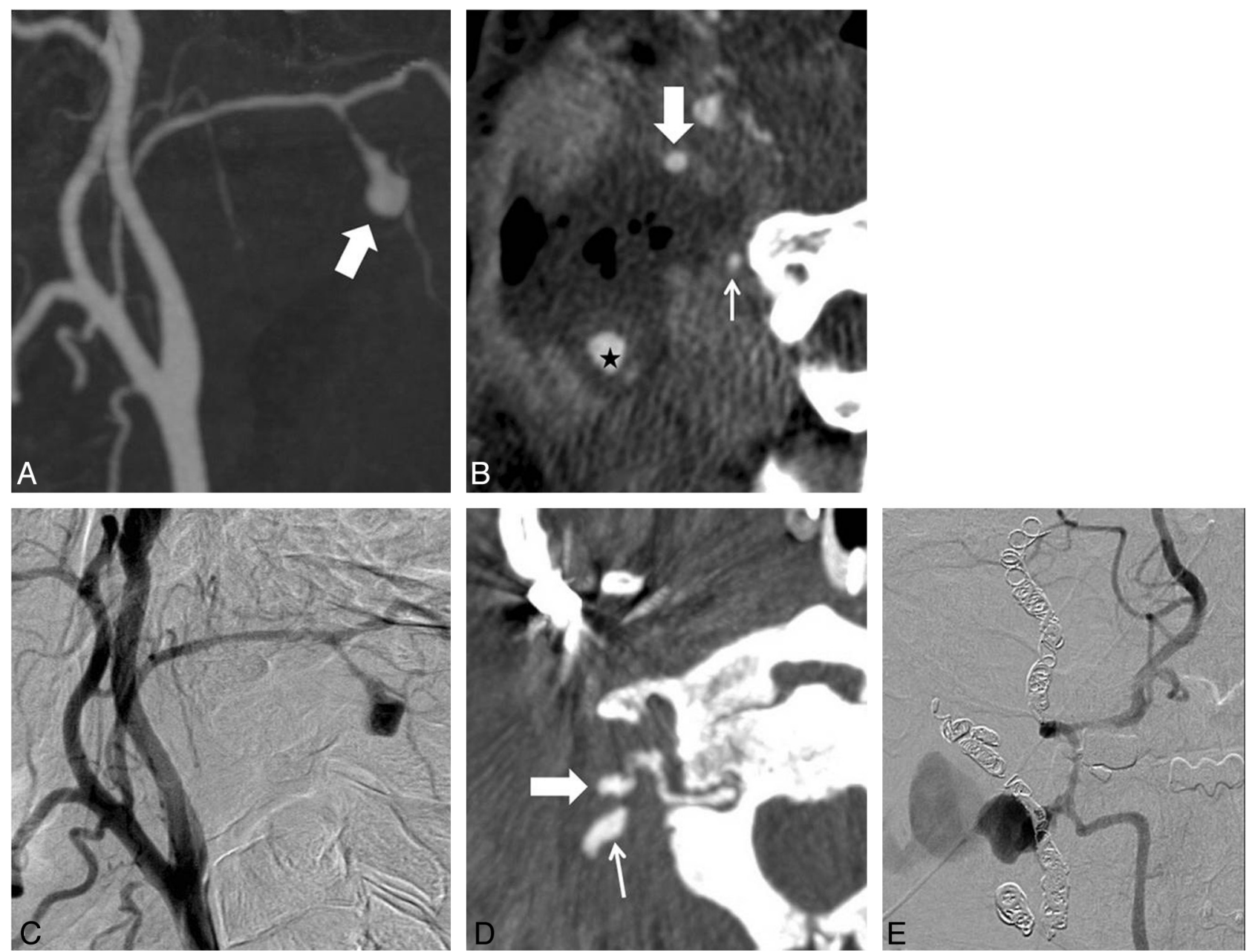

FIG 2. A 42-year-old man with a history of nasopharyngeal cancer. A, MIP CTA image shows a pseudoaneurysm (arrow) at the right occipital artery. $B$, Oblique reformatted image shows the pseudoaneurysm surrounded by necrotic tissue (star). Half of the circumference of the right internal carotid artery (large arrow) is exposed to the necrotic tissue, and the unexposed vertebral artery (small arrow) is close to the necrotic margin. C, DSA confirms the pseudoaneurysm of the occipital artery. The pseudoaneurysm is embolized with coils. At 64 days after treatment, another blowout occurs. D, Oblique reformatted CTA image shows a newly developed pseudoaneurysm (large arrow) with extravasation from the right vertebral artery (small arrow). E, DSA confirms the diagnosis.

Table 1: CTA findings in 29 patients with carotid blowout syndrome

\begin{tabular}{lccc}
\hline Imaging Finding & All CTA & First CTA & Second CTA \\
\hline Necrosis & & & \\
Percentage (ratio) & $94 \%(31 / 33)$ & $93 \%(27 / 29)$ & $100 \%(4 / 4)$ \\
$95 \% \mathrm{Cl}$ & $0.79-0.99$ & $0.77-0.99$ & $0.54-1.00$ \\
Viable perivascular tumor & & & \\
Percentage (ratio) & $67 \%(22 / 33)$ & $66 \%(19 / 29)$ & $75 \%(3 / 4)$ \\
$95 \% \mathrm{Cl}$ & $0.50-0.80$ & $0.47-0.80$ & $0.29-0.97$ \\
Pseudoaneurysm & & & \\
Percentage (ratio) & $58 \%(19 / 33)$ & $59 \%(17 / 29)$ & $50 \%(2 / 4)$ \\
$95 \% \mathrm{Cl}$ & $0.41-0.73$ & $0.40-0.75$ & $0.15-0.85$ \\
Contrast extravasation & & & \\
Percentage (ratio) & $30 \%(10 / 33)$ & $24 \%(7 / 29)$ & $75 \%(3 / 4)$ \\
$95 \% \mathrm{Cl}$ & $0.17-0.47$ & $0.12-0.42$ & $0.29-0.97$ \\
Exposed artery & & & \\
Percentage (ratio) & $73 \%(24 / 33)$ & $69 \%(20 / 29)$ & $100 \%(4 / 4)$ \\
$95 \% \mathrm{Cl}$ & $0.56-0.85$ & $0.51-0.83$ & $0.54-1.00$ \\
\hline
\end{tabular}

fore CTA and died 1 and 11 days, respectively, after treatment. Two of the 4 patients who refused PAO died from hemorrhagic shock within 1 day, and the other 2 patients died 20 and 32 days, respectively, later. All patients had PAO according to the CTA
Table 2: Interobserver agreement of CTA findings in 29 patients with carotid blowout syndrome

\begin{tabular}{lcc}
\hline \multicolumn{1}{c}{ Imaging Finding } & $\boldsymbol{\kappa}$ & $\mathbf{9 5 \%} \mathrm{Cl}$ \\
\hline Necrosis & 0.785 & $0.38-1.00$ \\
Viable perivascular tumor & 0.864 & $0.68-1.00$ \\
Pseudoaneurysm & 0.942 & $0.83-1.00$ \\
Contrast extravasation & 0.932 & $0.80-1.00$ \\
Exposed artery & 0.807 & $0.60-1.00$ \\
\hline
\end{tabular}

findings, including even 1 patient with negative DSA findings. One patient had conservative treatment and was lost to follow-up after 190 days.

\section{Patient Outcomes}

Five (17.5\%) of the 29 patients had rebleeding 52 to 162 days after the first CTA. Two had received conservative treatment, and 3 patients had received PAO after the first CBS. Four had CTA studies that showed new and different segmental exposed arteries. The survival curves of the 3 groups of patients with CBS are shown in Fig 3. The median survival time was more than 365 days in group $1(n=9), 141$ days in group $2(n=15)$, and 18 days in 
Table 3: Comparison of the diagnosis between the 23 studies of CTA and conventional angiography in 20 patients with carotid blowout syndrome

\begin{tabular}{|c|c|c|c|c|c|c|c|c|c|c|}
\hline & \multicolumn{10}{|c|}{ CTA } \\
\hline & \multicolumn{2}{|c|}{ Pseudoaneurysm } & \multicolumn{2}{|c|}{ Contrast Extravasation } & \multicolumn{2}{|c|}{ Viable Perivascular Tumor } & \multicolumn{2}{|c|}{ Necrosis } & \multicolumn{2}{|c|}{ Exposed Artery } \\
\hline & Positive & Negative & Positive & Negative & Positive & Negative & Positive & Negative & Positive & Negative \\
\hline Positive results on DSA & 17 & 1 & 6 & 1 & 5 & 0 & 0 & 0 & 0 & 0 \\
\hline Negative results on DSA & 0 & 5 & 4 & 12 & 11 & 7 & 23 & 0 & 20 & 3 \\
\hline
\end{tabular}

Survival Functions After 1st CTA

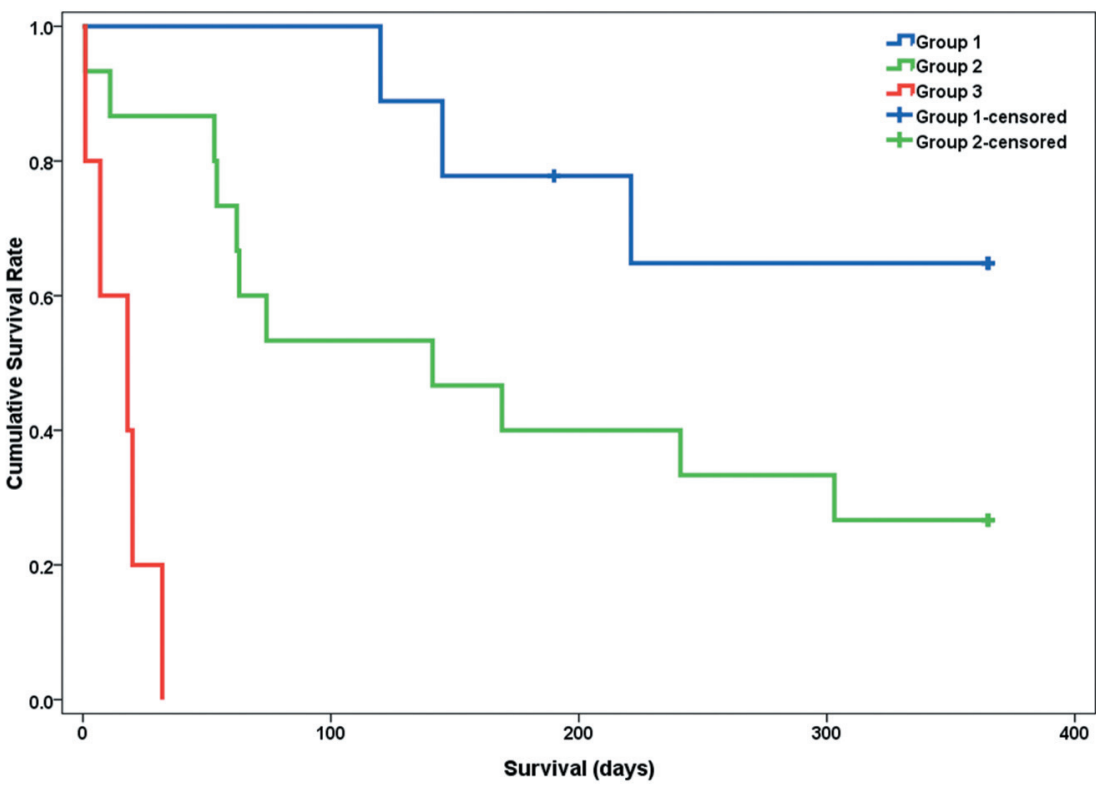

FIG 3. Kaplan-Meier survival curves with pair-wise log-rank test show group 1 (blue, no pseudoaneurysm/extravasation/exposed artery on CTA) has the best survival rate, followed by group 2 (green, with pseudoaneurysm/extravasation/exposed artery on CTA and treated by permanent artery occlusion), and the worst survival is noted in group 3 (red, with pseudoaneurysm/extravasation/exposed artery on CTA but not treated by permanent artery occlusion).

group $3(n=5)$. There were significant differences in survival time between group 1 and group $3(P<.001)$ and between group 2 and group $3(P<.001)$. The survival duration of group 1 tended to be better than in group 2 but did not reach statistical significance $(P=.056)$. There was no significant difference in survival duration between patients with $(n=19$; median survival duration, 145 days) and without ( $n=10$; median survival duration, 74 days) visible viable tumors $(P=.349)$, between patients with $(n=$ 18; median survival duration, 145 days) and without $(n=11$; median survival duration, 74 days) radical neck dissection, between patients with ( $n=25$; median survival duration, 141 days) and without ( $n=4$; median survival duration $>365$ days) irradiation $(P=.207)$, and between patients with ( $n=22$; median survival duration, 120 days) and without ( $n=7$; median survival duration $>365$ days $)$ chemotherapy $(P=.247)$. Only 2 patients had no necrosis, and they both survived for more than 1 year.

\section{DISCUSSION}

In our study, we found that CTA has a high accuracy for the diagnosis of impending CBS. Pseudoaneurysm, contrast extravasation, and exposed arteries on CTA were the 3 main indicators for aggressive treatment in patients with impending CBS. In patients with impending CBS with these 3 indicators, those treated with urgent PAO usually had a better outcome than those who received no or delayed treatment.

In our series, only patients with impending CBS underwent CTA, and the percentage of those with contrast extravasation was not high $(24.1 \%$ on initial CTA and $30 \%$ on all CTA). CTA showed contrast extravasation in 9 of 21 pseudoaneurysms and 10 of 26 exposed arteries. Among the 10 contrast extravasations that were shown on CTA, only 6 were identified on DSA, and 1 contrast extravasation was shown on DSA but not on CTA. This finding might be the result of intermittent bleeding and/or different timing of CTA vs DSA. Pseudoaneurysm was the most consistent finding between DSA and CTA. One pseudoaneurysm was shown on DSA but not on CTA, and this patient's DSA was done with delay. This result suggests that CTA is comparable to DSA in the detection of pseudoaneurysm in impending CBS. Necrosis and exposed arteries were the 2 most common CTA findings. An artery surrounded by necrotic tissue is prone to the development of a pseudoaneurysm and bleeding. ${ }^{26}$ DSA is essentially a study of the lumen of the vessel, and when extraluminal necrosis is present and is exposing the segmental artery, the lumen may appear angiographically normal. In our series, all pseudoaneurysms and extravasations were shown on an exposed arterial segment, and exposed arteries could only be detected by CTA, not by DSA. We suggest that an exposed artery is the most significant indicator on CTA for patients with CBS. DSA cannot detect an exposed artery and, in addition, might underestimate the extent of the lesion for PAO.

The overall incidence of recurrent CBS has been reported to be $26 \%$ and is mostly the result of progressive disease or treatment failure. ${ }^{27}$ The incidence of recurrent CBS has been described as similar in patients who have undergone PAO or endovascular stent placement. ${ }^{16,17}$ In our series, the rate of recurrent CBS was approximately $17.5 \%$, which is less than that in other published reports. We performed PAO according to CTA findings in the first CBS, and this might decrease the rate of treatment failure and occlude the vessel to a more accurate extent in our series. We recommend CTA to depict an exposed artery in patients with impending CBS, if possible.

CTA can noninvasively demonstrate the current status of diseased vessels without stroke risk in patients with episodic but clinically stable CBS, and prognostic information is rapidly avail- 
able. CTA can define groups of patients who had bleeding but do not demonstrate the 3 indicators for urgent management (group 1). In this group, the outcome was relatively good even without $\mathrm{PAO}$, and we believe that treatment of patients with impending CBS can be guided appropriately by CTA findings.

The survival duration was significantly better in group 1 than in group 3, indicating that patients without the 3 indicators on CTA had better outcomes than those with such indicators. The survival time was significantly better in group 2 than in group 3, indicating that the outcome in patients with impending CBS with these 3 indicators who received PAO was better than in those who received no or delayed treatment. The survival time was better in group 1 than in group 2, though the difference was not statistically significant. This finding indicates that PAO can improve the survival time in patients with these 3 indicators, or the progression of disease may cause new vascular lesions or death in both groups. A question that warrants further study is whether PAO of a normal-appearing artery very close to necrotic tissue can prevent bleeding in the future.

There were some limitations of our study that should be considered. This study was retrospective, and the number of patients was small because impending CBS is not a common disease. We did not perform $3 \mathrm{D}$ rotational angiography, which might have decreased the sensitivity of DSA. In addition, diagnosis of the subtle exposed arteries or small pseudoaneurysms on CTA is time consuming and experience dependent. Finally, we did not include patients with acute CBS who required immediate $\mathrm{PAO}$; thus, the results can be only applied to patients who are relatively stable hemodynamically.

\section{CONCLUSIONS}

CTA demonstrates imaging findings not available with DSA that have potentially useful prognostic information for patients with impending CBS. Contrast extravasation, pseudoaneurysm, and exposed artery were all associated with poorer outcomes, with an exposed artery being the most significant predictor. Endovascular treatment should be performed as soon as possible in patients with impending CBS with these findings. If the above findings are absent, then conservative treatment can result in acceptable outcomes.

\section{ACKNOWLEDGMENTS}

Thanks to National Science Council (NSC) support for the Center for Dynamical Biomarkers and Translational Medicine, National Central University, Taiwan (NSC 101-2911-I-008-001).

Disclosures: Adam Huang-RELATED: Grant: National Science Council, Taiwan, ${ }^{*}$ Comments: Government research funds; UNRELATED: Patents (planned, pending or issued): Pending patent applications: We have one U.S. patent application and 2 Taiwanese patent applications for removing bone from CT angiography imaging. ${ }^{*}$ Money paid to institution.

\section{REFERENCES}

1. Chaloupka JC, Putman CM, Citardi MJ, et al. Endovascular therapy for the carotid blowout syndrome in head and neck surgical patients: diagnosis and managerial considerations. AJNR Am JNeuroradiol 1996;17:843-52

2. Maran AGD, Amin M, Wilson JA. Radical neck dissection: a 19-year experience. J Laryngol Otol 1989;103:760-64

3. Fonkalsrud EW, Sanchez M, Zerubavel R, et al. Serial changes in arterial structure following radiation therapy. Surg Gynecol Obstet 1977;145:395-400

4. Ernemann U, Herrmann C, Plontke S, et al. Pseudoaneurysm of the superior thyroid artery following radiotherapy for hypopharyngeal cancer. Ann Otol Rhinol Laryngol 2003;112:188-90

5. Chaloupka JC, Roth TC, Putman CM, et al. Recurrent carotid blowout syndrome: diagnostic and therapeutic challenges in a newly recognized subgroup of patients. AJNR Am J Neuroradiol 1999;20:1069-677

6. Moore OS, Klarlan M, Sigler LS. Factors influencing the safety of carotid ligation. Am J Surg 1969;118:666-68

7. Citardi MJ, Chaloupka JC, Son YH, et al. Management of carotid artery rupture by monitored endovascular therapeutic occlusion (1988-1994). Laryngoscope 1995;105:1086-92

8. Dandy WE. Intracranial aneurysm of the internal carotid artery cured by operation. Ann Surg 1938;107:654-59

9. Dandy WE. Internal carotid arterial aneurysms in the carotid canal: diagnosis and treatment. Arch Surg 1942;45:521-33

10. Fox AJ, Viñuela F, Pelz DM, et al. Use of detachable balloons for proximal artery occlusion in the treatment of unclippable cerebral aneurysms. J Neurosurg 1987;66:40-46

11. Chaloupka JC, Putman CM. Endovascular therapy of surgical diseases of the cranial base. Clin Plast Surg 1995;22:417-50

12. Lesley WS, Chaloupka JC, Weigele JB, et al. Preliminary experience with endovascular reconstruction for the management of carotid blowout syndrome. AJNR Am J Neuroradiol 2003;24:975-81

13. Mathis JM, Barr JD, Jungreis CA, et al. Temporary balloon test occlusion of the internal carotid artery: experience in 500 cases. AJNR Am J Neuroradiol 1995;16:749-54

14. Standard SC, Ahuja A, Guterman LR, et al. Balloon test occlusion of the internal carotid artery with hypotensive challenge. AJNR Am J Neuroradiol 1995;16:1453-58

15. Dare AO, Chaloupka JC, Putman CM, et al. Failure of the hypotensive test during temporary balloon test occlusion of the internal carotid artery to predict delayed hemodynamic ischemia after therapeutic carotid occlusion. Surg Neurol 1998;50:147-56

16. Warren FM, Cohen JI, Nesbit GM, et al. Management of carotid "blowout" with endovascular stent grafts. Laryngoscope 2002; 112:428-33

17. Simental A, Johnson JT, Horowitz M. Delayed complications of endovascular stenting for carotid blowout. Am J Otolaryngol 2003;24:417-19

18. Kim HS, Lee DH, Kim HJ, et al. Life-threatening common carotid artery blowout: rescue treatment with a newly designed self-expanding covered nitinol stent. Br J Radiol 2006;79:226-31

19. Gaba RC, West DL, Bui JT, et al. Covered stent treatment of carotid blowout syndrome. Semin Intervent Radiol 2007;24:47-52

20. Chang FC, Lirng JF, Luo CB, et al. Patients with head and neck cancers and associated postirradiated carotid blowout syndrome: endovascular therapeutic methods and outcomes. J Vasc Surg 2008;47:936-45

21. Becker KJ, Baxter AB, Bybee HM, et al. Extravasation of radiographic contrast is an independent predictor of death in primary intracerebral hemorrhage. Stroke 1999;30:2025-32

22. Goldstein JN, Fazen LE, Snider R, et al. Contrast extravasation on CT angiography predicts hematoma expansion in intracerebral hemorrhage. Neurology 2007;68:889-94

23. Kim J, Smith A, Hemphill JC 3rd, et al. Contrast extravasation on CT predicts mortality in primary intracerebral hemorrhage. AJNR Am J Neuroradiol 2008;29:520-25

24. Li N, Wang Y, Wang W, et al. Contrast extravasation on computed tomography angiography predicts clinical outcome in primary intracerebral hemorrhage: a prospective study of 139 cases. Stroke 2011;42:3441-46

25. Demchuk AM, Dowlatshahi D, Rodriguez-Luna D, et al. Prediction of haematoma growth and outcome in patients with intracerebral haemorrhage using the CT-angiography spot sign (PREDICT): a prospective observational study. Lancet Neurol 2012;11:307-14

26. Chin SC, Jen YM, Chen CY, et al. Necrotic nasopharyngeal mucosa: an ominous MR sign of a carotid artery pseudoaneurysm. AJNR Am J Neuroradiol 2005;26:414-16

27. Chaloupka JC, Roth TC, Putman CM, et al. Recurrent carotid blowout syndrome: diagnostic and therapeutic challenges in a newly recognized subgroup of patients. AJNR Am J Neuroradiol 1999;20:1069-77 